\title{
Comparative Study on Mode-Selection and Tuning Principle of Several Birefringent Filters
}

\section{Shan Dujuan}

Department of Precision Instruments, School of Mechanical and Instrumental Engineering, Xi'an University of Technology, Xi'an, China

\section{Email address:}

791079934@qq.com

\section{To cite this article:}

Shan Dujuan. Comparative Study on Mode-Selection and Tuning Principle of Several Birefringent Filters. Science Discovery. Vol. 5, No. 2, 2017, pp. 144-150. doi: 10.11648/j.sd.20170502.19

Received: April 7, 2017; Accepted: April 20, 2017; Published: May 4, 2017

\begin{abstract}
Bireftingent filters play an important role in many fields such as laser technology, etc. This paper describes the mode-selection and tuning principle of several sets of new birefringent filters. Including PBS-HWP combination, PBS-QW combination, PBS-LN crystal combination and PBS-QWPs combination. The advantages and disadvantages of these bireftingent filters are analyzed, mainly about the convenience and laser structure, which are in good agreement with experiment results.
\end{abstract}

Keywords: Birefringent Filter, Lasers, Frequency Tuning

\section{几种双折射滤光片选模及调谐原理的对比研究}

\section{山杜娟}

机械与精密仪器工程学院精密仪器系, 西安理工大学, 西安, 中国

邮箱

791079934@qq. com

摘要: 双折射滤光片在激光技术领域扮演极其重要的角色。本文详述了几组新型双折射滤光片的选模及调谐原理（包 括PBS-二分之一波片组合、PBS-石英光楔、PBS-铌酸锂晶体、PBS-双四分之一波片），分析了这些双折射滤光片在调 谐便宜性、激光器结构等方面的优缺点, 对双折射滤光片的优化设计和实际应用具有理论指导意义。

关键词: 双折射滤光片, 激光器, 频率调谐

\section{1. 引言}

双折射滤光片作为光波长调谐元件，由于调谐方便、 插入损耗小等优点, 被广泛应用在天文光学系统和激光器 件等技术领域中。众所周知, 双折射是晶体光学中的重要 现象之一, 当一束光经过一块双折射晶体时, 将分裂为 2 束偏振方向不同的光。由于它们的传播速度不同, 响应产 生不同的相位延迟。近年来, 利用双折射晶体制作的滤光 片作为调光元件广泛应用于激光器内外。固体激光器存在 增益的空间烧孔效应, 激光器往往以多纵模振荡, 所以要
输出高质量的基横模单纵模, 选模是重中之重。而选模方 法多种多样, 如短腔法、扭转模腔、环形腔、标准具和双 折射滤光片等。频率可调谐也是激光器的主要评价指标之 一, 进行激光频率窄调谐的方法主要有压电效应调谐、温 度调谐和旋转标准具调谐等。多数情况采用双折射滤光片 来实现激光频率的宽调谐。所以双折射滤光片即可以进行 模式选择, 又可完成频率调谐, 近年来广泛应用于固体激 光器系统内。

以往对双折射滤光片的研究, 大多数集中在分析和考 虑其温度不敏感性、透过率、透过宽带、色散特性等特性 
上 [1]-[4], 但专门对比研究其调谐特性的很少讨论。本 文主要基于全固态激光器中的选模和调谐问题, 对比研究 几种新型双折射滤光片的选模和调谐原理, 对于双折射滤 光片的优化设计和实际应用具有理论指导意义。

\section{2. 研究内容及意义}

频率可调作为激光器的主要评价指标之一, 近年来实 现激光频率的宽调谐主要采用双折射滤光片 [5]。文章对 比分析了几组新型双折射滤光片的选模与调谐原理以及 各自的优缺点。首先以全固态Nd: YAG激光器为例, 对偏振 分光棱镜 (PBS) -二分之一波片组合的双折射滤光片的模 式选择原理及频率调谐原理进行了详细分析; 又因 (PBS) 一双四分之一波片组合的双折射滤波片不仅调谐范围广而 且非常有利于激光器的小型化, 亦对其进行了赘述。

文章对几组新型双折射滤光片选模及调谐原理的对 比分析, 对于全固态单频可调谐激光器的设计及应用有重 要的理论指导意义。为后续研究大频差可调谐双频固体激 光器奠定基础, 以应用于绝对距离干涉测量。

\section{3. 传统双折射滤光片的选模与调谐}

\section{1. 石英晶体的特性}

文中几组双折射滤光片中的双折射晶体均是用石英 晶体制作而成。石英晶体具有双折射效应, 属于正单轴晶 体。正单轴晶体的折射率曲面是一个双层曲面, 它是有一 个半径为 $n_{o}$ 的球和一个以 $x_{3}$ 轴为旋转轴旋转椭球构成的。 球面对应为 $\mathrm{o}$ 光的折射率曲面, 旋转粗球表示的是 $\mathrm{e}$ 光的折 射率曲面。正单轴晶体的折射率曲面在主轴截面上的截线 [6]如图1所示。

其中 $x_{3}$ 轴为光轴, 当与 $x_{3}$ 轴成夹角为 $\varphi$ 的波法线 $\mathrm{k}$ 与折射率曲面相交时, 得到长度为 $n_{o}$ 和 $n_{e}(\varphi)$ 的矢径, 它 们分别是相应于 $\mathrm{k}$ 方向的两个特许线偏振光的折射率, 其 中 $n_{e}$ 与 $\varphi$ 的关系式为:

$$
n_{e}(\varphi)=\frac{n_{o} n_{e}}{\sqrt{n_{o}^{2} \sin ^{2} \varphi+n_{e}^{2} \cos ^{2} \varphi}}
$$

可以看出, 当波法线方向 $\mathrm{k}$ 与光轴的夹角 $\varphi$ 发生变化 时, 对应的e光折射率 $n_{e}$ 也会发生变化。随着夹角 $\varphi$ 的增 大, $\mathrm{o}$ 光和 $\mathrm{e}$ 光的折射率差逐渐增大, 当夹角 $\varphi=90^{\circ}$ 时, $\mathrm{o}$ 光和e光的折射率差达到最大。

\section{2. 选模与调谐原理}

双折射滤光片选模是通过在腔内引入选择性损耗, 使 欲选纵模的损耗最小, 而其他纵模的损耗较大, 即加大各 纵模间净增益差异, 那么在激光形成过程中, 起振并得到 放大的是净增益较大的那个单纵模。如图2所示, 传统的 双折射滤光片 (BF) 由布儒斯特片和双折射晶体组成, 光 波通过布儒片 (BP) 成为线偏振光, 这里记作 $\mathrm{p}$ 偏振光; $\mathrm{p}$ 偏振光经过双折射晶体 (BC) 时产生双折射效应, (图3
所示为晶体的双折射现象) 分解为偏振方向互相垂直的o 光和e光; 光波往返通过 $\mathrm{BC}$ 后, $\mathrm{o}$ 光、 $\mathrm{e}$ 光间产生的相位差 为 $[7]$ 。

$$
\varphi=k \delta=\frac{2 \pi}{\lambda} \cdot \Delta n \cdot 2 l
$$

式中, $k$ 为波矢量, $\delta$ 为光程差, $\lambda$ 为光波长, $\Delta n$ 为o 光、 $\mathrm{e}$ 光的折射率差, $l$ 为 $\mathrm{BC}$ 的通光长度。当且仅当相位 差为 $2 \pi$ 整数倍, 即

$$
\varphi=\frac{2 \pi}{\lambda} \cdot \Delta n \cdot 2 l=2 m \pi
$$

时, 光波的偏振状态才不发生改变仍为 $p$ 偏振光。满足此 条件的光波再次通过BP时由于损耗较小在腔内优先起振; 而其他不满足条件的光波便不再是 $\mathrm{p}$ 偏振光, 再次通过 $\mathrm{BP}$ 时由于损耗过大而被抑制掉。由上式 (3) 可以推倒出双 折射滤光片透射峰的频率为

$$
v=\frac{c m}{2 \Delta n \cdot l}
$$

进而得到相邻两透射峰的频率间隔即双折射滤光片的自 由光谱范围 $\left(f_{F S R}\right)$ 为

$$
f_{F S R}=v_{m+1}-v_{m}=\frac{c}{2 \Delta n \cdot l}
$$
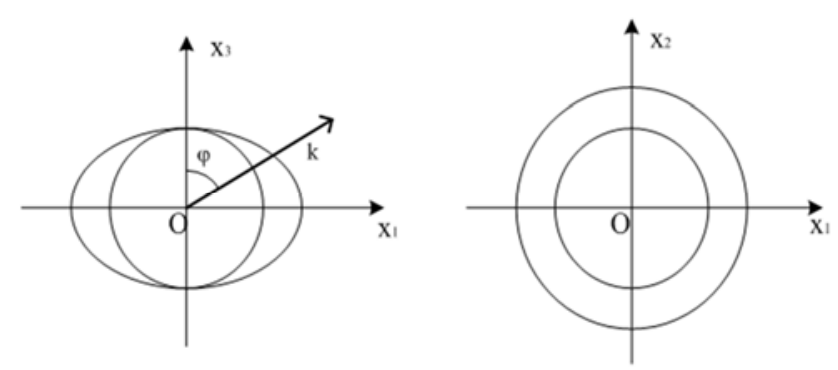

图1 正单轴晶体折射率曲面。

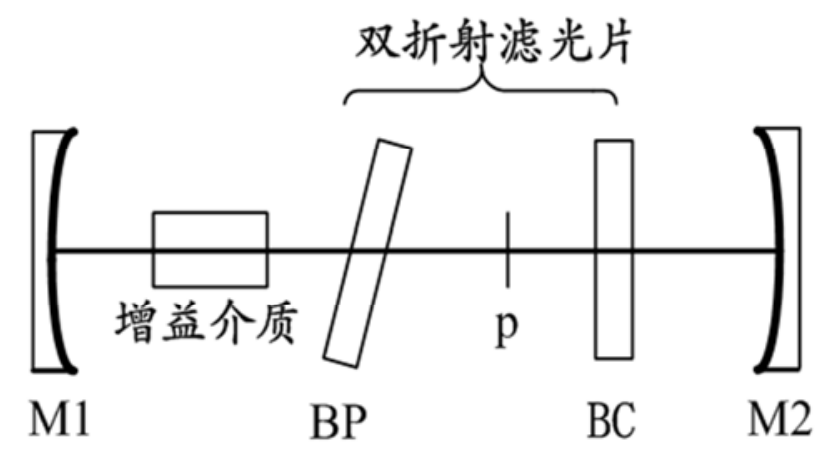

图2 含双折射滤光片的激光谐振腔。 


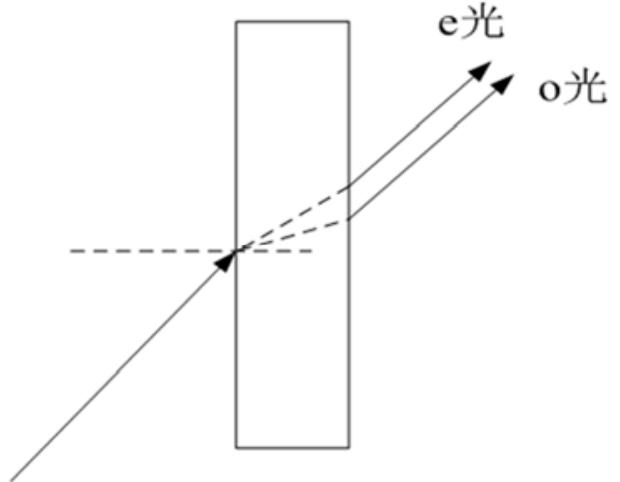

图3 晶体的双折射现象。

图4中所示, (a) 为激光器的荧光线宽; (b) 为激 光器谐振腔的纵模序列; (c) 为双折射滤光片的透射率 曲线; (d) 为所选取的单纵模。当双折射滤光片的自由 光谱范围 $f_{F S R}$ 大于激光器的增益带宽 $\Delta v_{o s c}$, 并且两相邻 纵模在透射峰附近的损耗差值足够大, 同时满足以上两个 条件时, 激光便以单纵模形式振荡输出, 如图 $4(\mathrm{~d})$ 中 $v_{q}$ 。 当双折射滤光片的相对折射率差 $\Delta n$ 或者通光长度 $l$ 的变 化或者二者同时变化时, 会引起双折射滤光片的透射峰频 率和自由光谱范围的改变, 进而会使其透射率曲线在频率 轴上移动, 选出不同的单纵模, 如图4 (d) 中的 $v_{q+1}$ [8]。 说明双折射滤光片不仅具有选模能力而且能够实现频率 调谐。

特别的, 在频率调谐过程中, 会有两种情况:

第一种, 双折射滤光片两透射峰之间的距离即 $f_{F S R}$ 的 大小发生变化以致其在频率轴上不等间隔 (两透射峰频率 间隔发生变化）移动;

第二种, 双折射滤光片 $f_{F S R}$ 的大小不发生变化但透射 峰频率大小会变化, 以致其透射率曲线在频率轴上发生等 间隔移动，达到选频目的。

下文将以几组具体的新型双折射滤光片对这两种情 况进行详述。

(a)

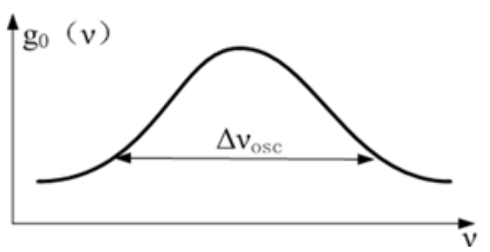

(b)

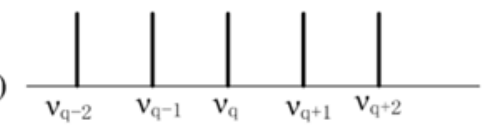

(c)

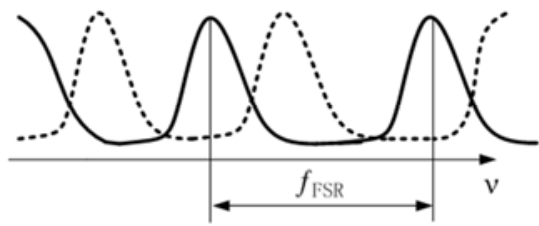

(d)

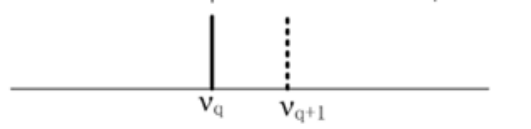

图4 单频激光选模与调谐原理图。

\section{4. 几种新型双折射滤光片}

下面依次介绍几组新型双折射滤光片PBS-二分之一 波片、PBS-石英光楔、PBS-铌酸锂晶体以及PBS-双四分之 一波片的选模、调谐原理, 及各自优缺点的对比分析。

\section{1. PBS-二分之一波片}

如图5所示, 首先偏振分光棱镜 (PBS) 较BP而言对p 光的透过率更高, 又光波往返通过二分之一波片后, $\mathrm{O} 、 \mathrm{e}$ 光间恰好产生 $2 \pi$ 整数倍的相位差。所以这种滤波片组合 具有更强的选模能力。 $\mathrm{p}$ 偏振光往返通过二分之一波片, o、 $\mathrm{e}$ 光间的光程差为:

$$
\varphi=\frac{4 \pi}{\lambda} \Delta n l
$$

满足

$$
\phi=2 m \pi
$$

的光频率在腔内形成振荡, 其他光频率由于损耗过大被抑 制掉。推算出此双折射滤光片的透射峰频率和自由光谱范 围分别为:

$$
\begin{gathered}
v=\frac{c m}{2 \Delta n \cdot l} \\
f_{F S R}=v_{m+1}-v_{m}=\frac{c}{2 \Delta n \cdot l}
\end{gathered}
$$

可见，当 $\Delta n 、 l$ 或者两者同时改变时，那么BF的透射 率曲线会在频率轴上移动, 以选取不同的单纵模, 实现频 率调谐。

以全固态Nd:YAG激光器为例分析这种组合的双折射 滤光片的调谐原理, 激光器结构如图6所示 [9], 由 (二极 管激光器） LD的尾纤 $\mathrm{OF}$ 出射的 $808 \mathrm{~nm}$ 泵浦光经过自聚焦透 镜SML汇聚到 Nd: $Y A G$ 晶体的左端面, Nd: $\mathrm{YAG}$ 的左端面镀有 对 $808 \mathrm{~nm}$ 抽运光高透和对 $1064 \mathrm{~nm}$ 振荡光高反的双色介质膜, $\mathrm{Nd}$ : YAG 的右端面镀有对 $1064 \mathrm{~nm}$ 光增透介质膜, OC为输出耦 合镜, 凹面镀有对 $1064 \mathrm{~nm}$ 激光部分透射的介质膜, 与 $\mathrm{Nd}$ : YAG的左端面形成平凹稳定腔。

若腔内不插入PBS及二分之一波片, 其输出为图 4 (b) 所示的纵模序列; 在双折射滤光片的作用下, 其输出为图 4 (d) 所示的单纵模; 通过倾斜二分之一波片实现频率的 调谐, 如图 4 (d) 所示选取不同单纵模。具体的调谐原理 如图7所示, $\varphi$ 为线偏光与波片晶轴的夹角, $\theta$ 为线偏光 与法线夹角, $\theta^{\prime}$ 为折射角, 自由光谱范围随晶轴倾斜角的 变化推倒如下:

$$
\begin{gathered}
\quad \Delta n=n_{e}(\varphi)-n_{o} \\
=\frac{n_{o} n_{e}}{\sqrt{n_{o}^{2} \sin ^{2} \varphi+n_{e}{ }^{2} \cos ^{2} \varphi}}-n_{o} \\
=\frac{n_{o} n_{e}}{\sqrt{n_{o}^{2} \cos ^{2} \theta+n_{e}{ }^{2} \sin ^{2} \theta}}-n_{o}
\end{gathered}
$$




$$
l=\frac{d}{\cos \theta^{\prime}}
$$

将（10）、（11）两式带入（9）可得晶轴倾斜角 $\boldsymbol{\theta}$ 与 自由光谱范围 $f_{F S R}$ 的直接关系为

$$
f_{F S R}=\frac{c \sqrt{1-\left(\frac{2 \sin \theta}{n_{o}+n_{e}}\right)^{2}}}{2 d\left(\frac{n_{o} n_{e}}{\sqrt{n_{o}^{2} \cos ^{2} \theta+n_{e}{ }^{2} \sin ^{2} \theta}}-n_{o}\right)}
$$

显然, $\theta$ 的改变直接引起双折射滤光片透射峰频率 $\boldsymbol{V}$ 以及自由光谱范围 $f_{F S R}$ 的大小。说明了此种双折射滤光片 调谐的可行性。

图8所示为输出激光频率改变量的频率随晶轴倾斜角 的变化仿真图, 图中显示: 当 $\theta$ 在 $-1.6^{\circ}$ 到 $0^{\circ}$ 变化时, 激光频率改变量呈减小趋势; 当 $\theta$ 在 $0^{\circ}$ 到 $1.6^{\circ}$ 变化时, 激光频率改变量呈增大趋势。实验结果表明 [9]: 腔内加 入此种双折射滤光片组合的 $\mathrm{Nd}$ : YAG激光器能以线偏振单 纵模稳定振荡; 当改变石英二分之一波片的晶轴倾斜角时, 可以实现 $1064 \mathrm{~nm}$ 单纵模激光的频率调谐, 振荡波长调谐量 为 $0.5 \mathrm{~nm}$, 相对应的频率调谐量为 $150 \mathrm{GHz}$, 这已经相当接 近Nd: YAG激光器的荧光线宽; $1064 \mathrm{~nm}$ 单频激光的频率和对 应功率随晶轴倾斜角的增大呈周期性变化, 每个周期内频 率的调谐量为 $150 \mathrm{GHz}$ 。

这种可调谐1064nm单频Nd: YAG激光器调谐范围大, 系 统结构简单, 调谐方便, 快速。可广泛应用于激光干涉测 量、激光雷达探测和激光光谱学等领域。

但是基于晶轴倾斜以改变 $l$ 的方法来实现频率调谐时, 倾斜角度特别小，对其的精确度不易控制。如果能够妥善 解决此问题, 那么这种激光器便可以作为微角度测量仪, 将会有很大前景。

\section{2. PBS-石英光楔}

如图9所示, $\mathrm{p}$ 偏振光往返通过二分之一波片, o、e光 间的光程差为:

$$
\varphi=\frac{4 \pi}{\lambda} \Delta n d
$$

\section{PBS}

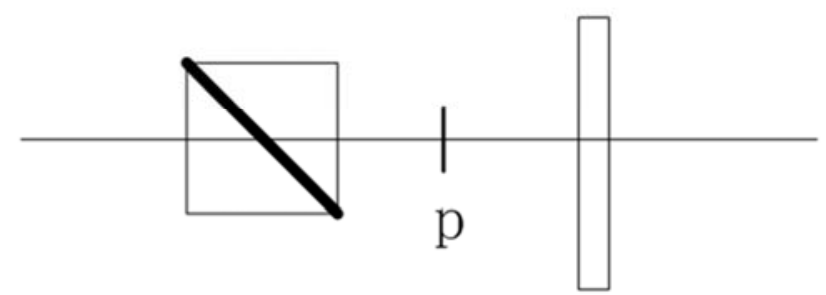

图5 PBS-二分之一波片组合的双折射滤光片。

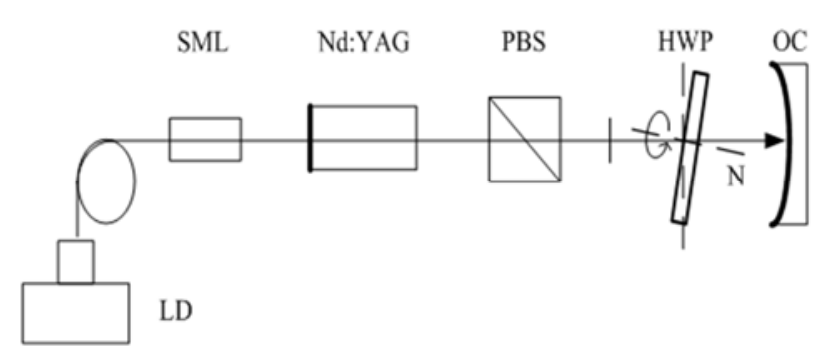

图6 LD抽运1064nm单频可调谐Nd:YAG激光器。

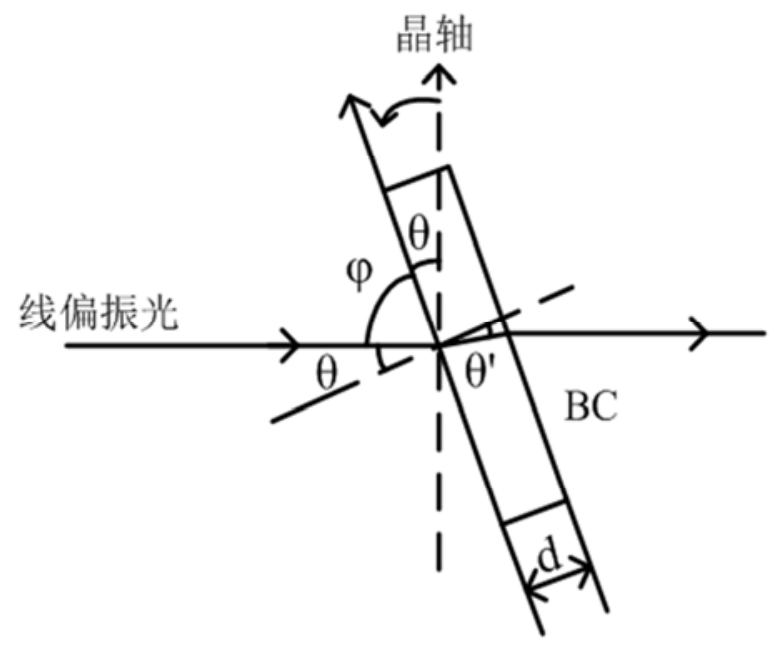

图7 晶轴倾斜频率调谐原理。

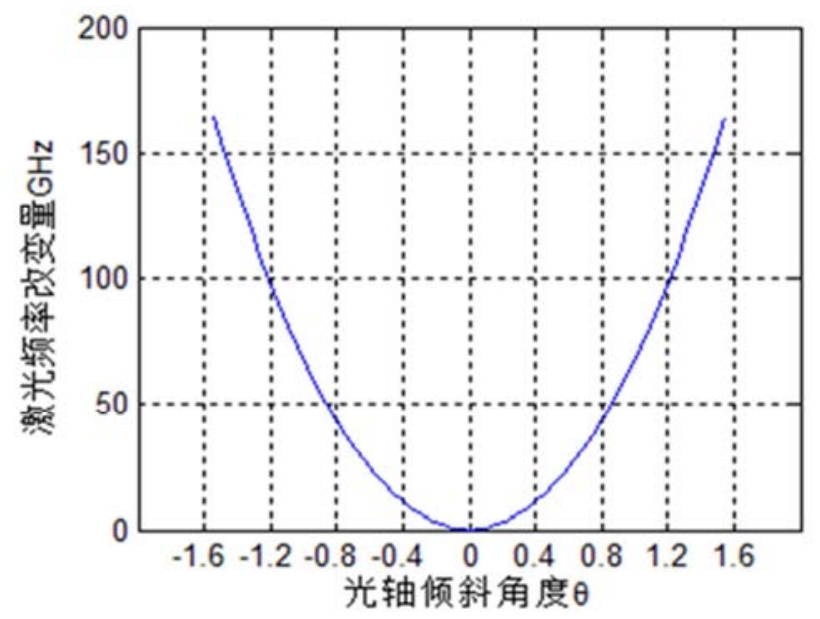

图8 晶轴倾斜角度 $\theta$ 在 $-1.6^{\circ} \sim 1.6^{\circ}$ 时频率变化规律。

其中, $d$ 为光楔的通光长度, $\Delta n$ 为石英光楔的折射率差。 当且仅当光程差 $\phi=2 m \pi$ 时, 光波的偏振态才不会发生改 变认为 $\mathrm{p}$ 偏光。满足条件的光频率在腔内形成振荡, 其他 光频率由于损耗过大被抑制掉。

推算出此双折射滤光片的透射峰频率和自由光谱范 围分别为:

$$
v=\frac{c m}{2 \Delta n \cdot d}
$$




$$
f_{F S R}=v_{m+1}-v_{m}=\frac{c}{2 \Delta n \cdot d}
$$

当 $\Delta n 、 d$ 或者两者同时改变时, 那么 $\mathrm{BF}$ 的透射率曲 线会在频率轴上移动, 以选取不同的单纵模, 实现频率调 谐。

可见，这种双折射滤波片组合与4.1. 滤波片的透射峰 频率和自由光谱范围大小相等, 但是频率可调是通过在垂 直光轴方向上移动石英晶体楔 [10] 以改变光楔的通光长 度 $d$ 来实现的, 这就比微角度的控制容易得多, 价格也要 低很多。若能精确控制微位移, 那么这种激光器的实验结 果可以用以设计微位移传感器。

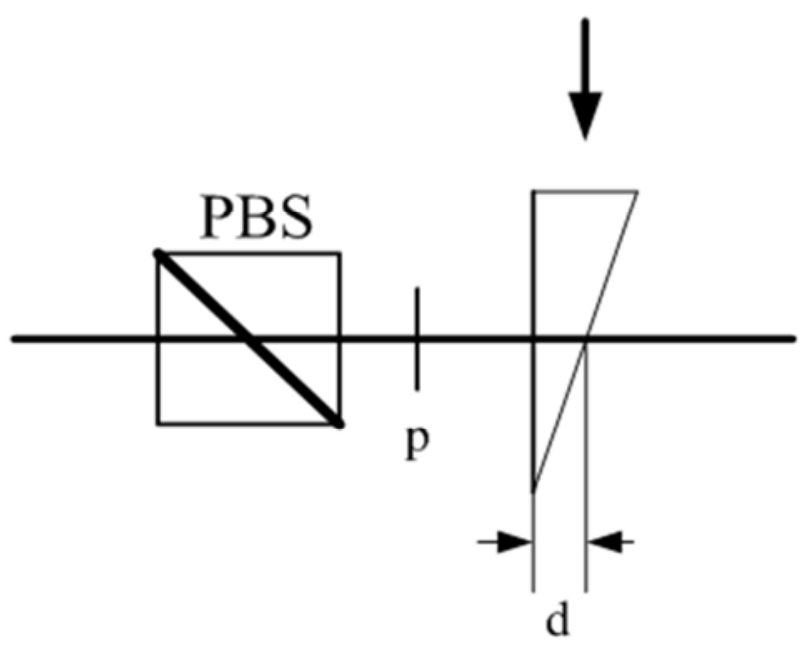

图9 PBS-光楔组合的双折射滤光片。

\section{3. 电光双折射滤光片: PBS-铌酸锂晶体LN}

如图10所示, 由物理光学知识知, 对于电光晶体LN, 当外加电场平行于光轴, 而光波沿垂直于光轴方向往返穿 过LN时, e光和o光这两个线偏振光之间产生受电场控制的 相位差为

$$
\varphi=\frac{4 \pi}{\lambda} \Delta n l+\frac{2 \pi n_{0}{ }^{3} r^{*} U}{\lambda} \frac{l}{d}
$$

式中, 等号右边第一项为自然双折射引起的相位延迟 量, 第二项为有外加电场后的感应双折射引起的相位延迟 量。其中, $\Delta n$ 为LN晶体的双折射率, $l$ 为光传播方向上的 晶体长度, $d$ 为电场方向上的晶体厚度, $n_{o}$ 为o光主折射 率, $r^{*}$ 为有效电光系数, $U$ 为沿光轴方向的外加电压。

当且仅当 $\phi=2 m \pi$ 时, 光波往返两次经过晶体LN后的 偏振状态才不会发生改变（仍为 $\mathrm{p}$ 偏振方向）, 这时再通 过PBS时的透射率最大, 受到的损耗最小, 可以优先起振。 而其他频率成分的光波偏振态发生改变受到抑制不能起 振。因此推出此双折射滤光片的透射峰频率 $\boldsymbol{V}$ 自由光谱范 围 (相邻两透射极大峰的频率间隔) $f_{F S R}$ 和损耗调制度 (相 邻两纵模的相位差） $\Delta \delta[8]$ 分别为:

$$
\begin{gathered}
v=\frac{m c}{2 \Delta n l+n_{o}{ }^{3} r^{*} U(l / d)} \\
f_{F S R}=\frac{c}{2 \Delta n l+n_{o}{ }^{3} r^{*} U(l / d)} \\
\Delta \delta=\frac{2 \pi \Delta n l+\pi n_{o}{ }^{3} r^{*} U(l / d)}{L}
\end{gathered}
$$

可见, $U$ 改变, $B F$ 的透射峰频率 $\boldsymbol{v}$ 和自由光谱范围 $f_{F S R}$ 均发生改变, 如图3中, BF 的透射率曲线在频率轴上 不等间隔 (两峰值间的频率间隔随 $U$ 发生变化) 移动。选 取不同单纵模, 实现调谐功能。

以此组合作为激光单纵模选择元件和频率调谐元件 设计Nd: YAG激光器, 结构简单、调谐方便、易于集成。

这种电光可调谐1064nm单频Nd: YAG的实验结果表明: 激光器能以线偏振单纵模稳定振荡, 当改变加在 $L N$ 晶体上 的横向电压时, 纵模激光振荡波长调谐量为 $0.474 \mathrm{~nm}$, 相 对应的频率调谐量为 $142.2 \mathrm{GHz}$ 。可广泛应用于激光干涉测 量、激光雷达探测和激光光谱学等领域。

但这种组合的双折射滤光片存在的缺点是, LN对电压 的承受能力有限, 所以所能加在其上的电压不能过大, 进 而限制了其频率调谐量。

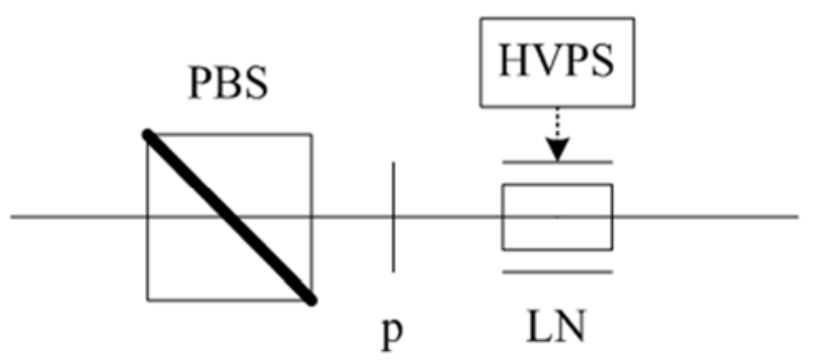

图10 PBS-LN组合的双折射滤光片。

\section{4. PBS-双四分之一波片} 差为:

光波往返通过双 $\lambda / 4$ 后, $\mathrm{o}$ 光、 $\mathrm{e}$ 光间产生的总的相位

$$
\varphi=\frac{8 \pi}{\lambda}\left(n_{e}-n_{o}\right) l+4(\alpha-\beta)
$$

其中, 第一项为自然双折射引起的相位延迟量, 第二 项为双 $\lambda / 4$ 波片快轴间夹角的存在引起的相位延迟量, 由 以下过程推知。

如下图12所示QWP1、QWP2为 $\lambda / 4$ 波片， $\alpha$ 为第一个 波片快轴与 $x$ 轴的夹角, $\beta$ 为第二个波片与 $x$ 轴的夹角。 腔内本振波在两个 $\lambda / 4$ 波片间任一截面内存在反向旋转 的两个圆偏光, 对不同的偏振态, $\lambda / 4$ 波片引起的附加相 移不同, 腔的本征波由原来的单频激光分裂成为具有不同 偏振状态的两个频率的本征态 $[11]$ 。

两 $\lambda / 4$ 波片的琼斯矩阵分别为 


$$
\begin{aligned}
J_{\alpha} & =\left[\begin{array}{ll}
\cos ^{2} \alpha+j \sin ^{2} \alpha & \sin \alpha \cos \alpha(1-j) \\
\sin \alpha \cos \alpha(1-j) & \sin ^{2} \alpha+j \cos ^{2} \alpha
\end{array}\right] \\
J_{\beta} & =\left[\begin{array}{ll}
\cos ^{2} \beta+j \sin ^{2} \beta & \sin \beta \cos \beta(1-j) \\
\sin \beta \cos \beta(1-j) & \sin ^{2} \beta+j \cos ^{2} \beta
\end{array}\right]
\end{aligned}
$$

光波往返通过双 $\lambda / 4$ 波片后的琼斯矩阵如下:

$$
J=J_{\lambda / 4}(\alpha) J_{\lambda / 4}(\beta) J_{\lambda / 4}(\beta) J_{\lambda / 4}(\alpha)
$$

$=\left[\begin{array}{cc}\cos 2(\alpha-\beta)+j \sin 2 \alpha \sin 2(\alpha-\beta) & j \cos 2 \alpha \sin 2(\alpha-\beta) \\ j \cos 2 \alpha \sin 2(\alpha-\beta) & \cos 2(\alpha-\beta)-j \sin 2 \alpha \sin 2(\alpha-\beta)\end{array}\right]$

由矩阵J的本征方程求得其相对应的本征值为:

$$
\left\{\begin{array}{l}
\lambda_{1}=\cos 2(\alpha-\beta)+j \sin 2(\alpha-\beta)=e^{2 j(\alpha-\beta)} \\
\lambda_{2}=\cos 2(\alpha-\beta)-j \sin 2(\alpha-\beta)=e^{-2 j(\alpha-\beta)}
\end{array}\right.
$$

分析可知, 由双 $\lambda / 4$ 波片快轴夹角对光波产生的附加 相位差为 [12]:

$$
\varphi_{1}=2(\alpha-\beta)-[-2(\alpha-\beta)]=4(\alpha-\beta)
$$

那么光往返通过双 $\lambda / 4$ 波片后产生的总的相位差为: $\varphi=\frac{2 \pi}{\lambda}\left(n_{e}-n_{o}\right) \cdot 4 d+\varphi_{1}=\frac{8 \pi}{\lambda}\left(n_{e}-n_{o}\right) d+4(\alpha-\beta)$

满足 $\phi=m 2 \pi$ 光频率的光在腔内形成振荡, 其他光波 由于损耗过大而被抑制掉, 推算出此双折射滤光片的透射 峰频率 $\boldsymbol{v}$ 和自由光谱范围（相邻两透射极大峰的频率间隔） $f_{F S R}$ 分别为:

$$
\begin{gathered}
v=\frac{c[m \pi-2(\alpha-\beta)]}{4 \pi l \Delta n} \\
f_{F S R}=\frac{c}{4 \Delta n l}
\end{gathered}
$$

式中, $\alpha-\beta$ 为双四分之一波片快轴的夹角。当改变 $\alpha-\beta$ 时, $\mathrm{BF}$ 透射峰的频率大小发生改变, 但是其自由光 谱范围并不改变。所以 $\alpha-\beta$ 改变, $\mathrm{BF}$ 的透射率曲线在频 率轴上等间隔移动, 即就是相邻两透射峰间隔不发生变化 但透射峰频率会发生变化, 以选取不同的单纵模。这种双 折射滤光片组合便属于上文中的第二种情况。 系为

由式 (27) 可知输出激光波长与双 $\lambda / 4$ 波片夹角的关

$$
\lambda=\frac{4 \pi d\left(n_{e}-n_{o}\right)}{m \pi-2(\alpha-\beta)}
$$

对 $\alpha-\beta$ 求导可知

$$
\Delta \lambda=\frac{8 \pi d\left(n_{e}-n_{o}\right)}{[m \pi-2(\alpha-\beta)]^{2}} \Delta(\alpha-\beta)
$$

结合 $v=c / \lambda$ 并对 $\alpha-\beta$ 求导可得

$$
\Delta \nu=-\frac{c}{2\left(n_{e}-n_{o}\right) d} \Delta(\alpha-\beta)
$$

图13所示为频率调节范围 $\Delta \boldsymbol{v}$ 随快轴夹角变化量 $\Delta(\alpha-\beta)$ 的变化曲线，可见二者之间成线性关系。

如若给第二片四分之一波片镀膜使之成为输出镜, 那 么可以大大简化激光器的结构, 非常有利于实现激光器的 小型化。

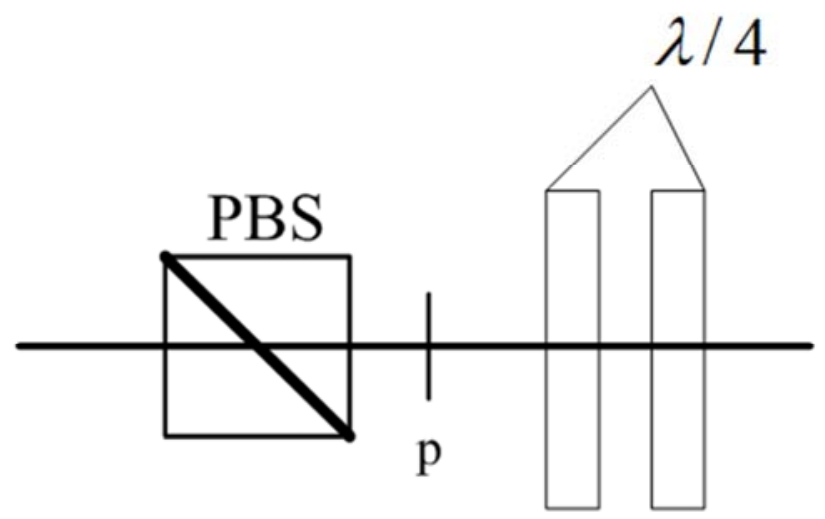

图11 PBS-双波片组合的双折射滤光片。

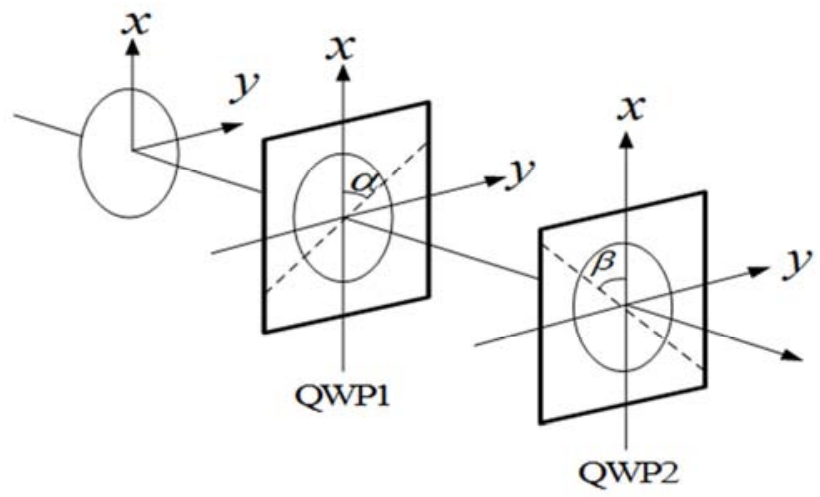

图12 双四分之一波片结构。

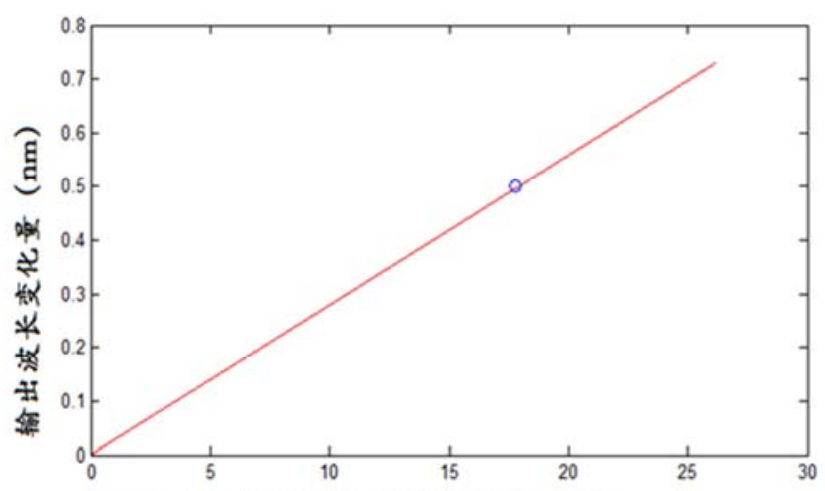

双四分之一波片快轴夹角的大小 (mrad)

图13 输出波长变化量与两波片夹角的对应关系。 


\section{5. 结论}

文章对各组合双折射滤光片的选模及调谐的可行性 进行了详细分析, 对于产生单频激光有着极其重要的作用。 与短腔法、扭转模腔、环形腔、标准具等相比, 利用双折 射滤光片进行纵模选择和频率调谐更加方便快捷, 也有利 于激光器的小型化。是实现单频可调谐激光器一种重要方 法, 具有实际的研究意义。

\section{参考文献}

[1] 高傲, 孔勇, 夏刚, 韩华, 王至金. 单轴双折射滤光片温度不 敏感的研究 $[J]$. 激光技术, 2016,40 (4) :476-478。

[2] Abde Rezzaq Halassi, Rachid Hamdi, Djalal Falih Bendimerad, Badr-Eddine Benkelfat. A novel synthsis approach for birefringent filters having arbitrarily amplitude transmittances[J]. Optics Communications, 2016, $265(2): 12-17$.

［3］穆廷魁, 李国华, 郝殿中. 宽调谐双折射滤光片最佳透射系 统研究 $[J]$. 激光技术, 2006, 30 (5)。
[4] 贾亚青, 朱晓农. 双折射滤光片的色散特性研究 [J]. 物理学 报，2004，53（9）。

[5] 焦明星, 冯其波, 王鸣, 刘君. 激光传感与测量 [M]. 北京: 科学出版社, 2014:89-90。

[6] 石顺祥, 王学恩, 刘劲松. 物理光学与应用光学 [M]. 西安: 西安电子科技大学出版社, 2008. 230-236。

[7] 高兰兰, 檀慧明. $\mathrm{LD}$ 葲浦的全固体单频激光技术 $[\mathrm{J}]$. 光机电 信息, 2002 (11)。

[8］邢俊红, 焦明星, 刘芸. 光电可调谐 $1064 \mathrm{~nm}$ 单频 $\mathrm{Nd}$ : YAG激光 器设计与实验研究. 中国激光 $[\mathrm{J}], 2014$, Vol. 41 (3) : 032007-1 0302007-6。

[9] 刘柳. LD抽运单频Nd: YAG激光器频率调谐技术研究 [D]. 西 安: 西安理工大学, 2016-06。

[10] 张书练. 正交偏振激光原理 $[\mathrm{M}]$. 北京: 清华大学出版社, 2005 。

[11] 张鹏. 激光二极管泵浦可调频差双频固体激光器 [J]. 红外 与激光工程, 2006, 35:129-133。

[12] 李磊. 激光二极管抽运频差可调谐双频固体激光器的研究 [J]. 物理学报, 2007, 5（56）:2663-266。 